\title{
Hemodynamic responses and serum nitrite concentration during uncontrolled hemorrhagic shock in normotensive and hypertensive rats
}

\author{
Majid Khazaei, Babak Barmaki, Ali Nasimi
}

\begin{abstract}
Background. We evaluated the effect of hypertension on hemodynamic responses and serum nitrite concentrations in normotensive (NT) and deoxycorticosteron acetate (DOCA)-Salt hypertensive (HT) rats.

Methods. Uncontrolled hemorrhagic shock was induced in NT and HT rats ( $\mathrm{n}=7$ each) by preliminary bleed of $25 \mathrm{ml} /$ $\mathrm{kg}$ followed by a $75 \%$ tail amputation. The mean arterial pressure (MAP), heart rate and serum nitrite were measured pre-hemorrhage and during hemorrhage.

Results. Changes in time-averaged MAP after hemorrhage were significantly greater in HT group than NT. After resuscitation, the HT rats failed to restore MAP to baseline level. Serum nitrite level in both groups was significantly increased during shock period. Survival rate of HT animals was lower than NT group, although it was not statistically significant. Conclusions. Marked reduction of MAP and less improvement after resuscitation suggested the less adaptation of cardiovascular system in HT animals which may interfere with management of these subjects during uncontrolled hemorrhagic shock.
\end{abstract}

Key words: hypertension, shock, hemorrhage, nitric oxide

Received: June 13, 2011; Accepted with revision: January 3, 2012; Available online: April 18, 2012 http://dx.doi.org/10.5507/bp.2012.006

Department of Physiology, Isfahan University of Medical Sciences, Isfahan, Iran Corresponding author:Majid Khazaei, e-mail:khazaei@med.mui.ac.ir

\section{INTRODUCTION}

Shock is a condition in which tissue perfusion is not capable to maintain normal tissue metabolism. Hemorrhagic shock is an emergency which can lead to hemodynamic instability, reduced tissue perfusion, cell hypoxia and finally death ${ }^{1}$.Trauma and gastrointestinal bleeding are the most common causes of hemorrhagic shock $^{1}$. During hemorrhagic shock, heart rate (HR) increases followed by bradycardia and in severe hypotension causes tachycardia ${ }^{2}$.

Hypertensive subjects are at risk of higher morbidity and mortality after hemorrhage ${ }^{3-5}$. It is indicated that baroreflex response to hemorrhage is impaired in spontaneous hypertensive rats ${ }^{3}$. In addition, a defect in hemodynamic responses after gastrointestinal hemorrhage in hypertensive subjects was reported ${ }^{6}$. Several circulating factors such as Nitric Oxide (NO) are involved in pathogenesis of various shockswhich modulate cardiovascular response to hemorrhagic shock ${ }^{7}$. NO is one of the most important endothelium-derived releasing factors which is synthesized by NO synthase ${ }^{8}$. There are three isoforms of NO synthase: constitutive, neuronal and inducible ${ }^{8}$.Excessive generation of $\mathrm{NO}$ is associated with vascular hyporeactivity to vasoconstrictive substances during shock ${ }^{7,9}$.

Since knowledge regarding the responses of hypertensive subjects to hemorrhagic shock has important implications for management of these patients, in this study we used uncontrolled hemorrhagic shock model in normotensive (NT) and deoxycorticosterone acetate (DOCA)Salt hypertensive (HT) rats to study the changes of the hemodynamic responses and serum nitrite concentration, the main metabolite of NO, during uncontrolled hemorrhagic shock and resuscitation.

\section{MATERIALS AND METHODS}

\section{Animal preparation}

Fourteen male wistar rats weighting 280-320 g (age between 10-12 weeks) were purchased from Pasteur institute of Iran. The animals were kept on 12-h light/dark cycle with $20-25{ }^{\circ} \mathrm{C}$ temperature and received standard rat chow and water ad libitum. All experiments were performed in accordance with the guidelines of the Animal and Human Ethical Committee of Isfahan University of Medical Sciences.

The animals were randomly divided into two groups: HT and NT ( $\mathrm{n}=7$ each). Hypertension was induced by subcutaneous injection of DOCA dissolved in almond oil $(30 \mathrm{mg} / \mathrm{kg}$ two times a week) in uninephrectomized rats and $\mathrm{NaCl} 1 \%$ and $\mathrm{KCl} 0.2 \%$ as drinking water ${ }^{10,11}$. In NT group, solvent of DOCA was injected and tap water used for drinking. Systolic blood pressure was recorded by tail cuff method every week. The rats with systolic blood pressure higher than $140 \mathrm{mmHg}$ were considered HT (ref. ${ }^{11}$ ).

\section{Shock protocol \\ After 8 weeks, the animals were anaesthetized with intraperitoneal injection of ketamin $(100 \mathrm{mg} / \mathrm{kg})$ and $\mathrm{xy}-$ lasine $(5 \mathrm{mg} / \mathrm{kg}$ ). A polyethylene catheter (PE-50) was inserted via right femoral artery for blood pressure and}




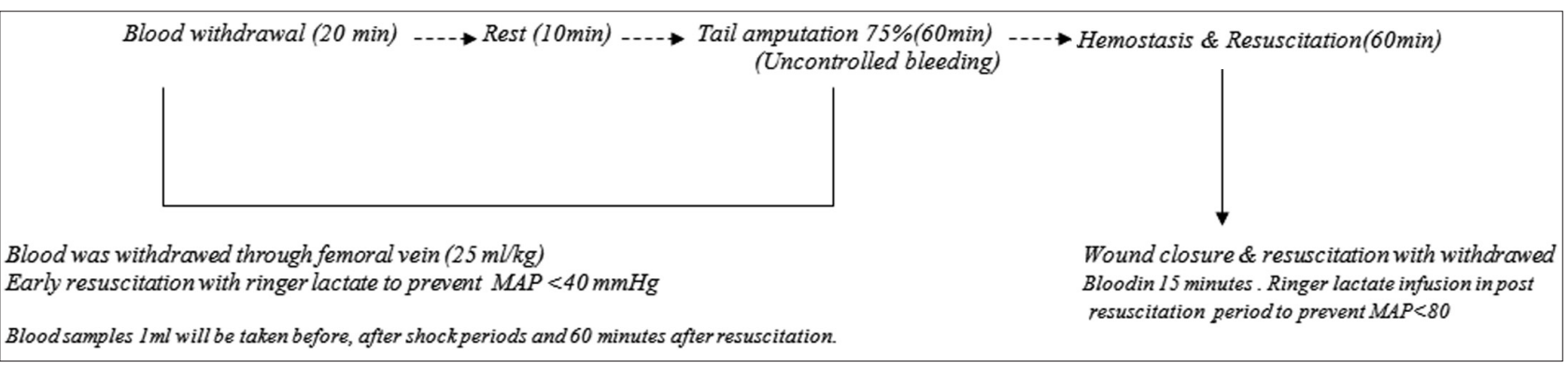

Fig. 1. Uncontrolled hemorrhagic shock protocol.

heart rate (HR) monitoring. Another PE-50 catheter was inserted via right femoral vein for blood withdrawal and sampling. Then, the animals were allowed $30 \mathrm{~min}$ rest period for stabilizing from surgery. Hemorrhagic shock was induced by withdrawing blood from femoral vein $(25 \mathrm{ml} / \mathrm{kg}$ ) for $20 \mathrm{~min}$ followed by $10 \mathrm{~min}$ rest period (Fig. 1)(ref. $\left.{ }^{3,12}\right)$. The shed blood was preserved for later re-infusion. Next, uncontrolled hemorrhage was added by amputation of $75 \%$ of the tail measured from the tip. The bleeding tail was directed into a container containing heparin to record cummulative hemorrhage volume. This phase was continued for 60 min (shock period). During this phase, ringer lactate was infused through femoral vein to maintain Mean Arterial Pressure (MAP) above $40 \mathrm{mmHg}$, if necessary (initial resuscitation phase). After shock period, tail wound was closed and initially shed blood re-infused during $15 \mathrm{~min}$. This phase was lasted 60 min and during this phase, MAP was maintained above $80 \mathrm{mmHg}$ with infusion of ringer lactate if necessary (post resuscitation phase)(ref. $\left.{ }^{13}\right)$. Volume of ringer lactate infusion in initial resuscitation and post resuscitation periods were recorded. Blood pressure and HR were recorded with a physiograph (Hugosachs electronic, Germany) and data analyzed with a windows compatible software. MAP and HR were determined at specific time points. Blood samples were taken before hemorrhage, immediately posthemorrhage and after resuscitation phase. Bloods were centrifuged and serums kept in $-70^{\circ} \mathrm{C}$ for further nitrite assay. At the end of experiment, catheters were removed and wounds closed. The animals were returned to their cages and allowed free access to water and food. Survival rate was determined every $12 \mathrm{~h}$ up to $72 \mathrm{~h}$.

\section{Serum nitrite measurement}

Serum nitrite concentrations were determined by conventional griess reaction method using available reagent (Promega, USA, Detection limit: $2.5 \mu \mathrm{mol}$ ) (ref. ${ }^{14}$ ).

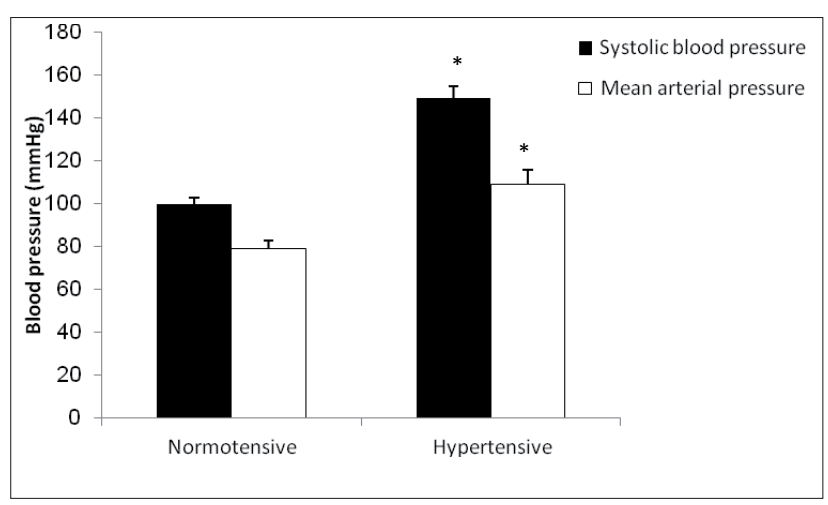

Fig. 2. Systolic blood pressure and MAP in NT and HT groups. *: $P<0.05$ compared with NT group. MAP: mean arterial pressure; NT: normotensive; HT: hypertensive.

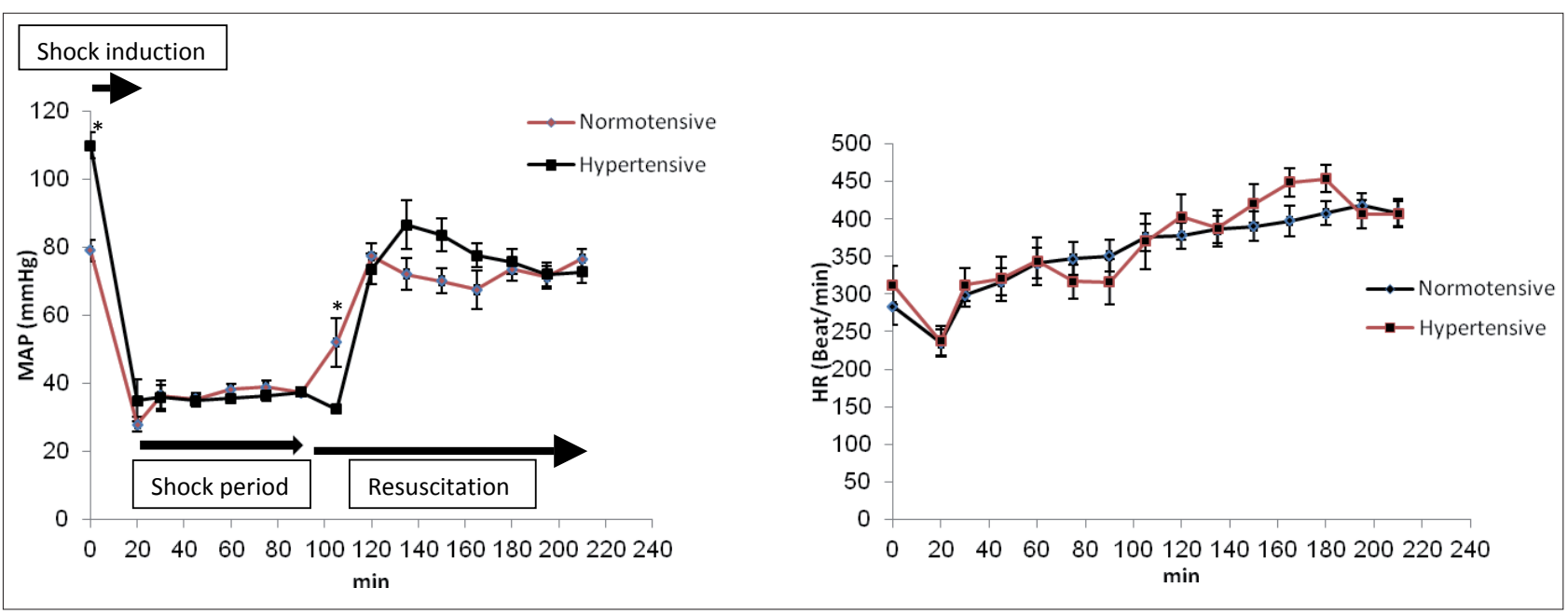

Fig. 3. Changes of MAP and HR during hemorrhagic shock and resuscitation in experimental groups.

*: $P<0.05$ compared with other group. MAP: mean arterial pressure; HR: heart rate. 
A: Shock induction

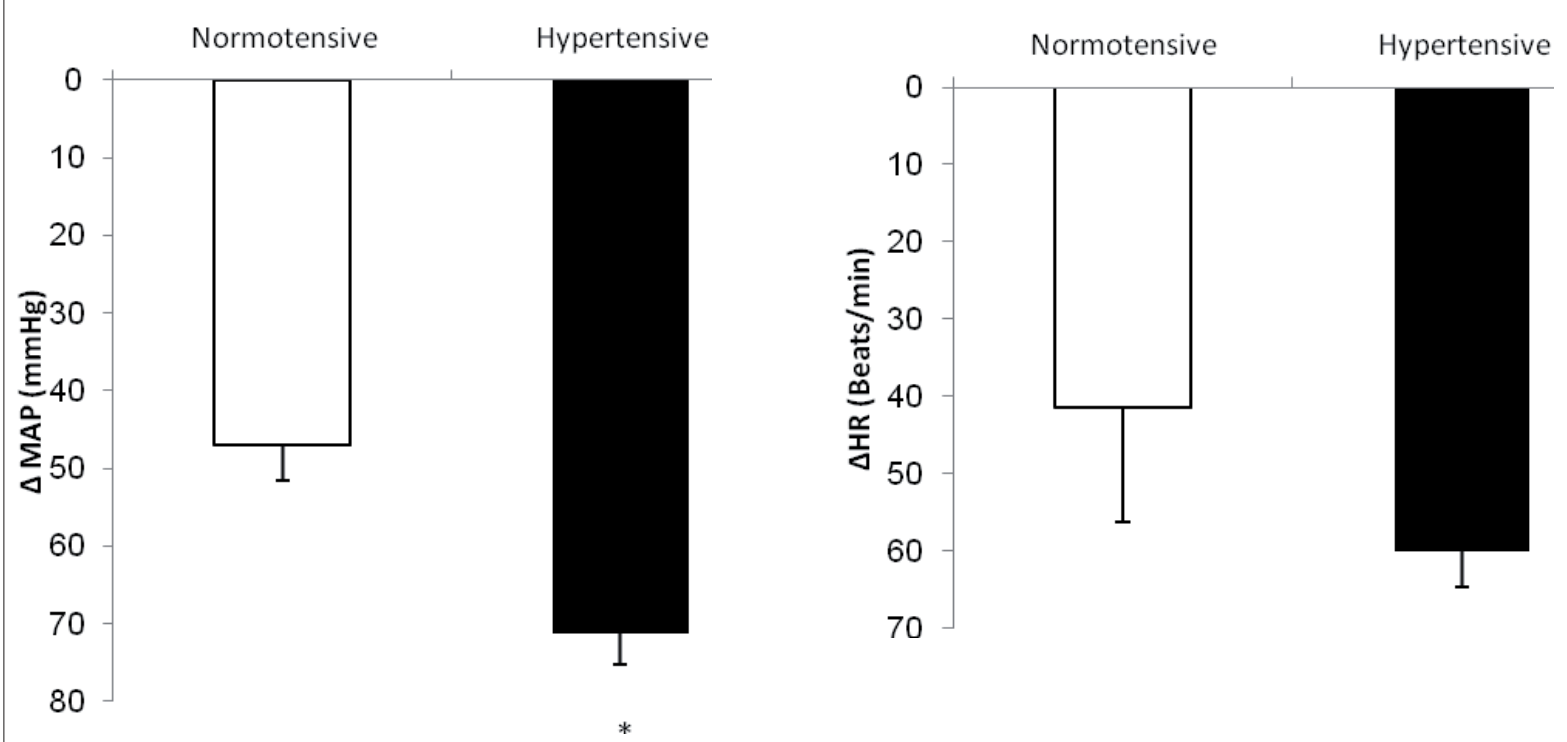

B: Resuscitation
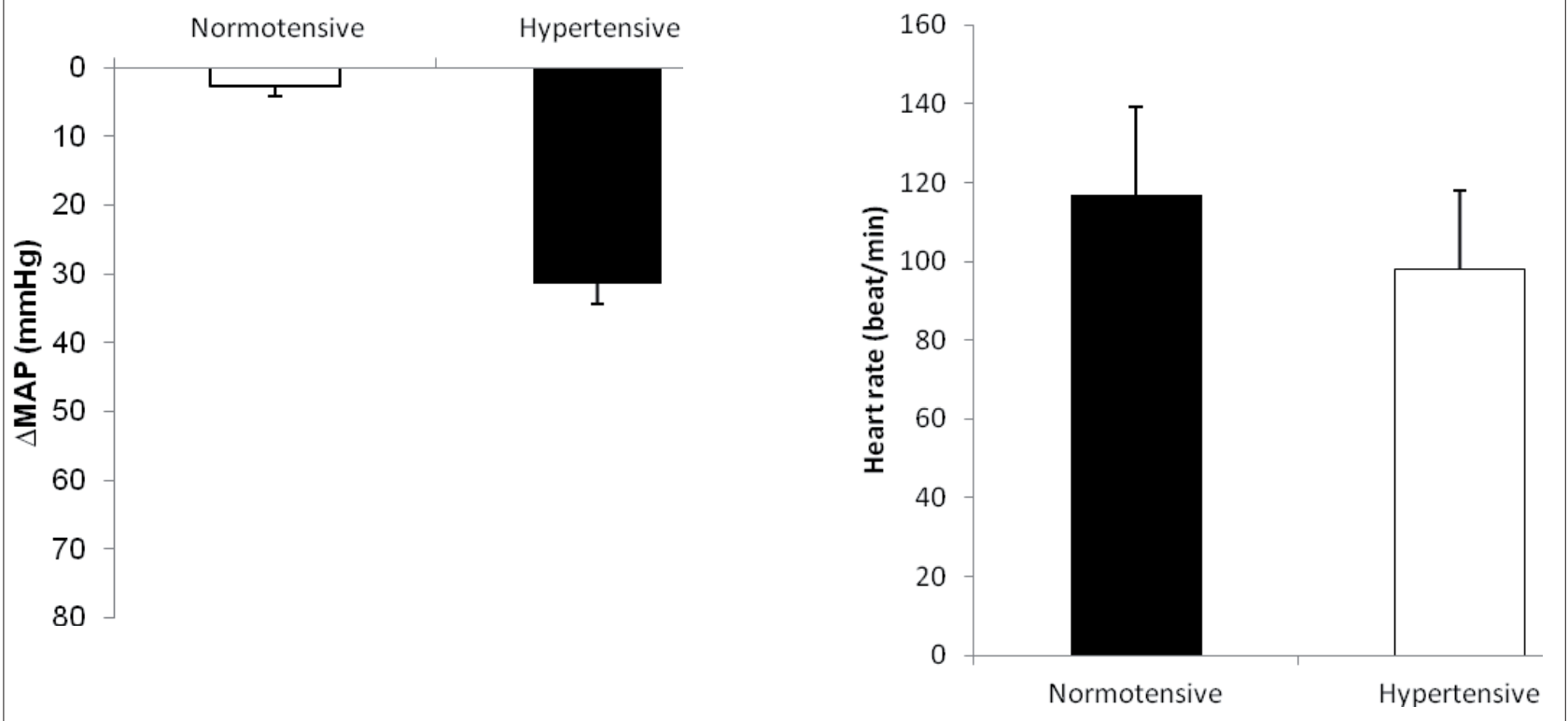

Fig. 4. Changes of MAP and HR after induction of uncontrolled hemorrhage (A) and resuscitation (B).

${ }^{*} P<0.05$ when compared with normotensive group. MAP: mean arterial pressure; HR: heart rate.

\section{Statistical analysis}

Repeated measure ANOVA followed by tukey's test was used for comparison of continuous variables. Comparison of data before and after shock was analyzed by paired t test. Data between two groups was analyzed by independent t test. Survival rate was evaluated by Chi square Fischer exact test. Bivariate correlation was calculated using pearson's correlation coefficient. $P \leq 0.05$ was considered statistically significant.

\section{RESULTS}

\section{Hemodynamic changes}

Systolic blood pressure and MAP of NT and HT groups were shown in Fig. 2. DOCA-Salt HT rats had significantly higher blood pressure than NT group $(P<0.05)$. Fig. 3 illustrates changes of MAP and HR of the NT and HT rats during hemorrhage and resuscitation. Blood withdrawal $(25 \mathrm{ml} / \mathrm{kg})$ via venous catheter for 20 min caused 


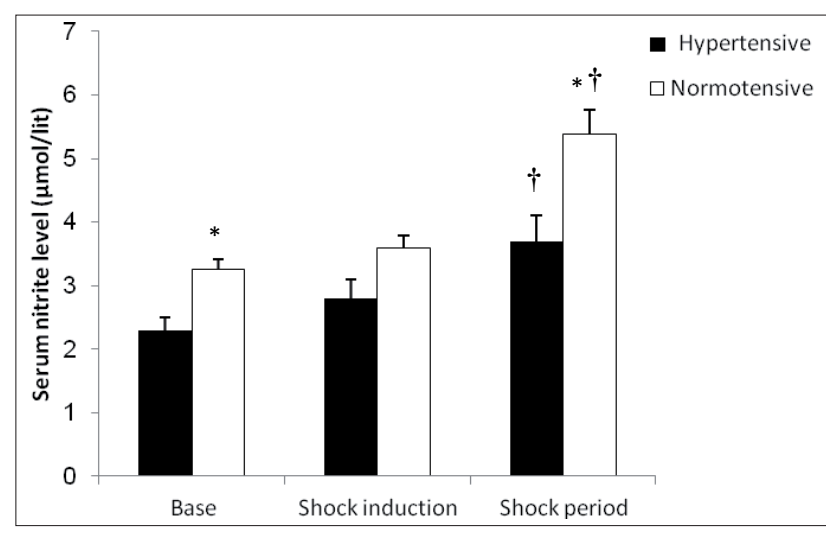

Fig. 5. Serum nitrite levels before shock (base), after shock induction and during shock period in experimental groups.

$*: P<0.05$ when compared with normotensive group.

$\dagger: P<0.05$ when compared with base and shock period.

a marked decrease in MAP in both NT and HT groups $(47 \pm 4.14 \mathrm{mmHg}$ vs. $74 \pm 4.22 \mathrm{mmHg}$, respectively; $P<0.05)$. HT animals experienced more reduction of MAP than NT group (Fig. 3,4A). During shock period, MAP was maintained around $40 \mathrm{mmHg}$ using ringer lactate solution infusion (Fig. 2). After resuscitation, MAP was increased in both groups, however, it was closer to basal level (prehemorrhage) in NT than HT group $(P<0.05)$ (Fig. 4B).

Baseline HR was slightly higher in HT group compared with NT $(P>0.05)$. Hemorrhage initially caused HR reduction in both groups Then, HR increased continuously during hemorrhage and resuscitation (Fig. 4A,B), however, there was no significant difference between groups.

\section{Serum nitrite measurement}

Fig. 5 illustrates serum nitrite concentration before hemorrhagic shock, after shock induction and at the end of shock period. Before hemorrhage, serum nitrite level in HT groups was significantly lower than NT $(P<0.05)$. Immediately after hemorrhage, serum nitrite was increased in both groups. At the end of shock period, serum nitrite concentration in both groups was higher than before hemorrhage $(P<0.05)$.

\section{Volume of tail bleeding and saline infusion during early resuscitation}

Volume of tail bleeding in HT group was higher than NT group, although it was not statistically significant ( $3.66 \pm 0.59$ vs. $2.07 \pm 0.53 \mathrm{ml} ; P<0.05)$. The initial fluid volume required to maintain MAP around $40 \mathrm{mmHg}$ (early resuscitation phase) in HT rats was higher than NT rats $(19.4 \pm 2.58$ vs. $15.21 \pm 2.51 \mathrm{ml} ; P<0.05)$. Fluid volume infused in addition to shed blood in late resuscitation phase were not different between HT and NT groups $(5.50 \pm 1.23$ vs. $6.56 \pm 1.49 \mathrm{ml} ; P<0.05)$.

\section{Survival analysis}

Two out of seven HT rats were died during $4 \mathrm{~h}$ after experiment. Two out of seven HT and one out of seven NT rats were died during first $12 \mathrm{~h}$ after resuscitation.

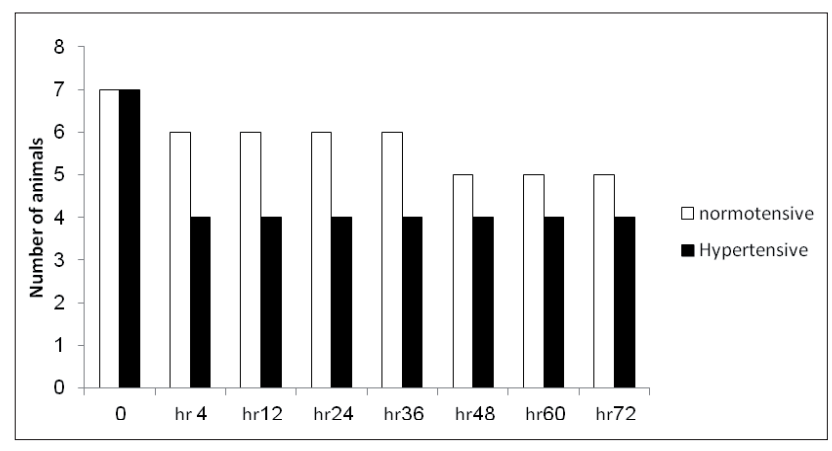

Fig. 6. Comparison of survival count between groups during 72 hours survival assay.

One out of seven NT rats was died after $48 \mathrm{~h}$. The rest of animals in each group were alive until 72 h (Fig. 6). By correlation analysis, we found that there is no correlation between serum nitrite concentration after resuscitation and mortality rate after $72 \mathrm{~h}(\mathrm{r}=0.10)$.

\section{DISCUSSION}

This study investigated the effect of uncontrolled hemorrhagic shock on hemodynamic responses and serum nitrite concentration in NT and HT rats.

Shock is a state of tissue hypoperfusion with hemodynamic instability. Our data showed that immediately post-hemorrhage, HT rats experienced more MAP reduction than NT rats as evidenced by larger drop in MAP. Moreover, during the resuscitation phase, MAP almost completely returned to basal level in NT group, while, the HT rats failed to restore MAP to baseline level. We also found that blood loss from tail cut (uncontrolled hemorrhage phase) and the initial fluid volume required to maintain MAP during early resuscitation phase in HT group was higher than NT, although it was not statistically significant. This condition means a hypotensive state for HT animals and could place them at risk of tissue ischemia and failure and may increase mortality rate in these animals. Previous studies demonstrated that hypertensive subjects are at risk of higher morbidity and mortality after blood $\operatorname{loss}^{3-5}$. In the present study, we showed that the survival rate of HT animals was lower than NT group. Marked reduction of MAP and less improvement of blood pressure after resuscitation may be partly responsible for higher mortality in HT animals.

Our observations are in parallel with other findings that reported HT rats as compared with NT group, had higher mortality rate after the same vascular injury, however, post-hemorrhage blood pressure and base excess were not different between groups ${ }^{3}$. Another study also demonstrated that hypertensive animals experienced more profound hemorrhagic shock compared with nor- 
motensive animals ${ }^{15}$. In this study, we used uncontrolled hemorrhagic shock model. In this model, in spite of catheter controlled hemorrhagic model, vascular injury (amputation of tail) results in continued blood loss ${ }^{3}$. Experimental and clinical studies indicated that there is a defect in hemodynamic and baroreflex responses after hemorrhage in hypertensive subjects ${ }^{6}$. Furthermore, hypertensive animals have greater tissue ischemia such as liver, skeletal muscles and brain than normotensive ${ }^{15}$.

Several circulating factors such as NO are involved in modulation of cardiovascular response to hemorrhagic shock $^{7}$. Excessive formation of NO may contribute to pathogenesis of various shock and vascular hyporeactivity in various shocks ${ }^{7,16,17}$. In the present study, serum nitrite level in HT animals was lower than NT group which may be the result of endothelial dysfunction in these animals ${ }^{18,19}$. In this study, hemorrhagic shock increased serum nitrite concentration in both groups. Activating of $\mathrm{NO}$ synthase during shock may be responsible forexcessive generation of $\mathrm{NO}\left(\mathrm{ref}^{20}\right)$. Furthermore, it is demonstrated that inducible form of NO synthase expressionisup-regulated in different organs during shock ${ }^{21,22}$. It was shown that inhibition of NO synthase in hemorrhagic shocked rats restored vasoconstrictive response to phenylephrine and angiotensin II ( ref. $^{20,23}$ ). Therefore, excessive formation of nitrite may increases reactive oxygen species and PGE2 which might result in organ damage ${ }^{24}$, although, in this study, we did not evaluate tissue ischemia during blood loss.

\section{CONCLUSION}

In conclusion, marked reduction of MAP during uncontrolled hemorrhagic shock. Less improvement of blood pressure after resuscitation, and higher fluid therapy during early resuscitation phase suggested the less adaptation of cardiovascular system in HT animals during blood loss which may interfere with management of these subjects. In addition, increased serum nitrite concentration during hemorrhagic shock may contribute to higher mortality of HT animals during severe hemorrhage.

\section{ACKNOWLEGMENT}

This study was supported by a grant from Isfahan University of Medical Sciences (grant number \# 387413).

\section{REFERENCES}

1. Angele MK, Schneider CP, Chaudry IH. Bench-to-bedside review: latest results in hemorrhagic shock. Crit Care 2008;12:218.

2. Secher $\mathrm{NH}$, Jacobsen J, Friedman DB, Matzen S. Bradycardia during reversible hypovolaemic shock: associated neural reflex mechanisms and clinical implications. Clin Exp Pharmacol Physiol 1992;19:733-43.

3. Sinert R, Spencer MT, Wilson R, Silverberg M, Patel M, Doty Cl, Sapan A, Baron B.J. The effect of hypertension on uncontrolled hemorrhage in a rodent model. Acad Emerg Med 2002;9:767-74.
4. Perdue PW, Watts DD, Kaufmann CR, Trask AL. Differences in mortality between elderly and younger adult trauma patients: geriatric status increases risk of delayed death. J Trauma 1998;45:805-10.

5. Milzman DP, Boulanger BR, Rodriguez A, Soderstrom CA, Mitchell KA, Magnant CM. Pre-existing disease in trauma patients: a predictor of fate independent of age and injury severity score. J Trauma 1992;32:236-43.

6. Radisavljevic Z. Hypertension-induced dysfunction of circulation in hemorrhagic shock. Am J Hypertens 1995;8:761-67.

7. Balaszczuk AM, Arreche ND, Mc LM, Arranz C, Fellet AL. Nitric oxide synthases are involved in the modulation of cardiovascular adaptation in hemorrhaged rats. Vascul Pharmacol 2006;44:417-26.

8. Forstermann U. Nitric oxide and oxidative stress in vascular disease. Pflugers Arch. 2010;459:923-39.

9. Gardiner SM, Kemp PA, March JE, Bennett T. Cardiac and regional haemodynamics, inducible nitric oxide synthase (NOS) activity, and the effects of NOS inhibitors in conscious, endotoxaemic rats. Br J Pharmacol 1995;116:2005-16.

10. Beswick RA, Zhang H, Marable D, Catravas JD, Hill WD, Webb RC. Long-term antioxidant administration attenuates mineralocorticoid hypertension and renal inflammatory response. Hypertension 2001;37:781-86.

11. Seifi B, Kadkhodaee M, Karimian SM, Zahmatkesh M, Xu J, Soleimani $M$. Evaluation of renal oxidative stress in the development of DOCAsalt induced hypertension and its renal damage. Clin Exp Hypertens 2010;32:90-97.

12. Xiao N, Wang XC, Diao YF, Liu R, Tian KL. Effect of initial fluid resuscitation on subsequent treatment in uncontrolled hemorrhagic shock in rats. Shock 2004;21:276-80.

13. Kentner R, Safar P, Prueckner S, Behringer W, Wu X, Henchir J, Ruemelin A, Tisherman SA. Titrated hypertonic/hyperoncotic solution for hypotensive fluid resuscitation during uncontrolled hemorrhagic shock in rats. Resuscitation 2005;65:87-95.

14. Nematbakhsh $M$, Khazaei $M$. The effect of estrogen on serum nitric oxide concentrations in normotensive and DOCA Salt hypertensive ovariectomized rats. Clin Chim Acta 2004;344:53-7.

15. Sinert R, Guerrero P, Quintana E, Zehtabchi S, Kim CN, Agbemadzo A, Baron $B J$. The effect of hypertension on the response to blood loss in a rodent model. Acad Emerg Med 2000;7:318-26.

16. Liu LM, Ward JA, Dubick MA. Hemorrhage-induced vascular hyporeactivity to norepinephrine in select vasculatures of rats and the roles of nitric oxide and endothelin. Shock 2003;19:208-14.

17. Liu LM, Dubick MA. Hemorrhagic shock-induced vascular hyporeactivity in the rat: relationship to gene expression of nitric oxide synthase, endothelin-1, and select cytokines in corresponding organs. J Surg Res 2005;125:128-36.

18. Gkaliagkousi E, Douma S, Zamboulis C, Ferro A. Nitric oxide dysfunction in vascular endothelium and platelets: role in essential hypertension. J Hypertens 2009;27:2310-20.

19. Levy AS, Chung JC, Kroetsch JT, Rush JW. Nitric oxide and coronary vascular endothelium adaptations in hypertension. Vasc Health Risk Manag 2009;5:1075-87.

20. Shirhan M, Moochhala SM, Kerwin SY, Ng KC, Lu J. Influence of selective nitric oxide synthetase inhibitor for treatment of refractory haemorrhagic shock. Resuscitation 2004;61:221-9.

21. McDonald M, Abdelrahman M, Cuzzocrea S, Thiemermann C. Tyrphostin reduces the organ injury in haemorrhagic shock: role of inducible nitric oxide synthase. Resuscitation 2003;58:349-61.

22. Anaya-Prado R, Toledo-Pereyra LH, Guo RF, Reuben J, Ward PA, Walsh $J$. The attenuation of hemorrhage-induced liver injury by exogenous nitric oxide, L-arginine, and inhibition of inducible nitric oxide synthase. J Invest Surg 2003;16:247-61.

23. Pieber D, Horina G, Sandner-Kiesling A, Pieber TR, Heinemann A. Pressor and mesenteric arterial hyporesponsiveness to angiotensin II is an early event in haemorrhagic hypotension in anaesthetised rats. Cardiovasc Res 1999;44:166-75.

24. Md S, Moochhala SM, Siew Yang KL, Lu J, Anuar F, Mok P, Ng KC. The role of selective nitric oxide synthase inhibitor on nitric oxide and PGE2 levels in refractory hemorrhagic-shocked rats. J Surg Res. 2005;123(2):206-14. 